\title{
Redshift drift in radially inhomogeneous Lemaître-Tolman-Bondi spacetimes
}

\author{
Romain Codur and Christian Marinoni* \\ Aix Marseille Univ, Université de Toulon, CNRS, CPT, Marseille, France
}

(Dated: December 17, 2021)

\begin{abstract}
We provide a formula for estimating the redshift and its secular change (redshift drift) in Lemaitre-TolmanBondi (LTB) spherically symmetric universes. We compute the scaling of the redshift drift for LTB models that predict Hubble diagrams indistinguishable from those of the standard cosmological model, the flat lambda cold dark matter $(\Lambda \mathrm{CDM})$ model. We show that the redshift drift for these degenerate LTB models is typically different from that predicted in the $\Lambda \mathrm{CDM}$ scenario. We also highlight and discuss some unconventional redshift-drift signals that arise in LTB universes and give them distinctive features compared to the standard model. We argue that the redshift drift is a metric observable that allows us to reduce the degrees of freedom of spherically symmetric models and to make them more predictive and thus falsifiable.
\end{abstract}

PACS numbers:

\section{INTRODUCTION}

The cosmological principle (CP) states that the universe is homogeneous and isotropic on large scales, i.e., invariant under translations and rotations around each comoving observer. Long regarded only as a philosophically appealing, if not logically necessary, convention on the symmetries of space [1, 2], it has now acquired the most scientifically legitimate status of a useful working hypothesis that can be questioned and verified by astronomical data [3-11]. The fact that the standard cosmic metric deduced from it, the FriedmannRobertson-Walker line element (FRW), is still practically indisputable, should not make us lose sight of the importance of challenging the standard paradigm and analysing the cosmological relevance of alternative spacetime proposals.

The phenomenology induced by cosmic inhomogeneities and their impact on the interpretation of past light cone data is still far from being satisfactorily understood [12]. Nonetheless, among the various inhomogeneous spacetimes, spherically symmetric and radially inhomogeneous models of the universe, the so-called Lemaittre-Tolman-Bondi (LTB) models [13-15] have being investigated in some details. They are widely regarded as interesting testing ground for challenging the $\mathrm{CP}$ or for non-canonical interpretations of cosmological observations [see 16, 17, for a review].

The popularity of the LTB metric models stems from the fact that they offer an alternative physical explanation for the Hubble diagram measured via Supernovae Ia (SNIa). The weakening of light from distant supernovae would be caused, in this inhomogeneous scenario, by radial spatial gradients in the rate of expansion and density of matter, rather than, as currently preferred, by a dark energy component forcing the second derivative of the cosmic scale factor $a(t)$ to be positive [18-25]. Indeed, since we have direct access only to data on our light-cone, it is challenging to disentangle temporal evolution in the scale factor from radial spatial variations. For example, it is possible to mimic the effect of dark energy by postulating that we live in a region of the universe under-

*Electronic address: christian.marinoni@cpt.univ-mrs.fr dense with respect to the average, which extends to the point in space where the acceleration/deceleration transition takes place (at redshift $z \sim 0.6$ ). Typically one invokes the hypothetical existence of a deep void, almost centered around us, with a radius that, depending on models, can be as big as the horizon ( $3 \mathrm{Gpc})$. Interestingly, LTB modelling of smaller size voids $(\sim 300 \mathrm{Mpc})$, which are compatible with tentative evidences about the existence of a local under density in the galaxy distribution [26, 27], has been recently advocated as a way to lessen the tension between local and early epochs measurements of the present day Hubble parameter $H_{0}$ [25, 28].

From the theoretical side, it has been argued that any spatially uniform data set can be misinterpreted by carefully tuning the functional degrees of freedom of an inhomogeneous LTB model [29]. This is ultimately due to the fact that this spacetime has fewer symmetries than the standard metric of the universe. For example, distance-redshift relations of the standard $\Lambda$ CDM models can be reconstructed with arbitrary precision using the freedom in the specification of the arbitrary boundary conditions of the LTB models [30]. Even if fine-tuned to reproduce the $\Lambda$ CDM Hubble diagram, the LTB models should retain their cosmological predictiveness when distance-independent observables are considered. It is thus of some interest to devise cosmological testing schemes that might resolve this degeneracy. In this paper we explore if and to what level this can be achieved by studying the temporal drift of the redshift [31,32].

The time variation of the redshift is a cosmological quantity that, being directly measurable from data, gives access to the evolution of the past-light cone in real time and in a modelindependent way [33]. It is thus a crucial observable for the so-called cosmographic approach to cosmology [34]. While the physics is straightforward, the observational implementation of this probe is challenging: dimensional arguments suggests that the signal is of order $\dot{z} \sim H(t)$ and thus very precise and stable observations over a period of several years are necessary to reach a signal to noise ratio of unity. Notwithstanding, the Extremely Large Telescope (ELT) [35, 36], the Square Kilometre Array (SKA) [37] and the Canadian Hydrogen Intensity Mapping Experiment (CHIME) [38] observational facilities promise to reach the necessary sensitivity.

Redshift drift data, once interpreted in the FRW framework, 
are expected to provide competitive constraints on the Hubble constant [39] as well as on some representative dark energy models [40 47]. The scaling of the redshift drift has also been investigated in alternative metric scenarios such as in arbitrary spacetimes [48, 49], in Stephani universes [50], in axially symmetric quasi-spherical Szekeres models [51] and backreaction models [52]. As far as the LTB model is concerned, the redshift drift has been calculated numerically by [53] although, as argued in [54], the intrinsic smallness of the redshift drift effect is a challenging factor for numerical codes, demanding careful architecture of non-naïve algorithms. An exact formula using observational coordinates has been proposed by [4] although it does not match numerical results, as claimed by [52]. Here we explore these issues and present an exact algebraic expression that provides the explicit dependence of the redshift drift on the structural parameters of the LTB metric in comoving coordinates.

The paper is organized as follows; in Sec. III we briefly review some key features of the LTB cosmological model and introduce our notations. In Sec. III we derive an explicit expression for the redshift of light as a function of the LTB comoving coordinates, as seen by a an observer sitting at the center of symmetry of the metric while in IV we provide the analytical expressions of its first and second time variations. Various potential systematic biases are discussed in Sec. V and applications to a specific LTB model are shown in Sec. VI Sec.VII provides summary and conclusion.

In the following, we present results in natural units $(c=1)$ and we refer to the standard $\Lambda$ CDM model, as the flat FRW spacetime which best fits the Planck18 data [55].

\section{THE LTB COSMOLOGICAL MODEL}

\section{A. Kinematics of light}

Universes with radial spatial inhomogeneities can be charted using spherical coordinates $x^{i} \equiv(\chi, \theta, \phi)$ that comove ( $U^{i} \equiv d x^{i} / d t=0$ ) with matter. It is convenient to set up the spatial origin $\left(x^{i}=0\right)$, the center of symmetry, at the observer position, and to choose the time coordinate $\left(x^{0} \equiv t\right)$ so that it measures the proper time of the comoving matter particles. The line element takes the form

$$
d s^{2}=d t^{2}-\alpha^{2}(t, \chi) d \chi^{2}-A^{2}(t, \chi)\left(d \theta^{2}+\sin ^{2} \theta d \phi^{2}\right)
$$

and we will refer to the two degrees of freedom $\alpha$ and $A$ as the radial and angular scale factor, respectively.

The geodesic motion of massive and massless particles for the central LTB observer is given by

$$
\frac{d k^{\mu}}{d \lambda}+\Gamma_{v \rho}^{\mu} k^{v} k^{\rho}=0
$$

where $\mu=0,1,2,3, k^{\mu}=d x^{\mu} / d \lambda$ is the tangent to the null geodesics and $\lambda \in \mathbb{R}$ is an affine parameter that describes the particles path. Because of the spherical symmetry, the geodesics are entirely determined by only two equations

$$
\begin{aligned}
& \frac{d k^{0}}{d \lambda}=-\alpha \dot{\alpha}\left(k^{1}\right)^{2} \\
& \frac{d k^{1}}{d \lambda}=-\frac{\alpha^{\prime}}{\alpha}\left(k^{1}\right)^{2}-2 \frac{\dot{\alpha}}{\alpha} k^{0} k^{1}
\end{aligned}
$$

where the overdot and a prime indicate a partial derivative with respect to the coordinates $t$ and $\chi$ respectively. Nullgeodesics are further characterized by the constraint $k^{\mu} k_{\mu}=0$, which supplies the additional condition

$$
k^{0}=-\alpha k^{1}
$$

where the sign convention stems from the hypothesis that the affine parameter and the radial coordinate $\chi$ are chosen to increases in the opposite direction of cosmic time, i.e. the equations are integrated backward on the past light cone of the observer. Moreover, since the affine parameter is determined up to a linear transformation, without loss of generality we set $\lambda=0$ at the observer position and set as boundary condition $k^{0}(\lambda=0)=-1$ in the chosen length units.

\section{B. Dynamics of dust}

The evolution of $\alpha$ and $A$ follows from imposing that the metric evolves in space and time according to the Einstein's field equations (EFE). For the purpose of our analysis, which deals with low-redshift phenomena, we will assume that the universe is in a dust dominated phase. Therefore the stress energy tensor describing the matter distribution, modelled as a perfect fluid, is $T^{\mu v}=\rho_{m}(t, \chi) U^{\mu} U^{v}$ where $U^{\mu}=\delta_{0}^{\mu}$ are the components of the 4-velocity-field of the fluid with respect to the chosen coordinate system and $\rho$ the invariant matter density seen by a comoving observer.

The EFE, supplemented by the cosmological member $\Lambda$, remove one metric degrees of freedom by imposing the constraint

$$
\alpha=A^{\prime} / \sqrt{1-k(\chi)}
$$

where $k(\chi)<1$ is an arbitrary function of the radial coordinate $\chi$ only, and a prime denotes a derivative with respect to this coordinate. This function describes the position dependent spatial curvature of the $t=$ const hypersurfaces. The function $A$ is determined via the equation

$$
\left(\frac{\dot{A}}{A}\right)^{2}+\frac{k}{A^{2}}=\frac{8 \pi G}{3}\left(\tilde{\rho}+\rho_{\Lambda}\right)
$$

where $\rho_{\Lambda}$ is the non-dilutive energy density associated with the cosmological constant $\Lambda$ and where we have defined the flat average density of matter

$$
\tilde{\rho}(t, \chi)=3 \frac{\int_{0}^{\chi} \rho_{m} A^{2} A^{\prime} d \chi}{A^{3}}
$$

as the non-local quantity satisfying the continuity equation (see Appendix A for details)

$$
\dot{\tilde{\rho}}+3 \frac{\dot{A}}{A} \tilde{\rho}=0 .
$$


This quantity must not be conceptually confused with (and it is in general quantitatively different from) the actual average density inside a shell of comoving coordinate $\chi$. Indeed, the averaging procedure is not carried out over the (spherical) volume element $d V=4 \pi A^{2} A^{\prime}(1-k(\chi))^{-1 / 2} d \chi$. Anyway, as the name suggests, the flat and the actual average densities of matter coincide in LTB models with $k(\chi)=0$. Moreover, in the absence of inhomogeneities, when the average density coincides with the local one, one finds that $\tilde{\rho}(t)=\rho_{m}(t)$. By inverting Eq. (8) one also finds the expression of the local matter density as a function of $\tilde{\rho}$

$$
\rho_{m}=\tilde{\rho}+\tilde{\rho}^{\prime} \frac{A}{3 A^{\prime}} .
$$

The advantage of introducing $\tilde{\rho}$ is that it brings the LTB formulas into closer formal analogy with those of the standard model of cosmology. This is shown explicitly by the equations (7), 9) or (11) and is also evident by inspecting the acceleration equation, which, in this notation, reads simply

$$
\frac{\ddot{A}}{A}=-\frac{4}{3} \pi G\left(\tilde{\rho}-2 \rho_{\Lambda}\right) \text {. }
$$

Finally, note that if there is no shell crossing during the motion of the spherically symmetric matter inhomogeneity, the integral on the numerator of the rhs of Eq. (8) is time independent.

Since Eq. 7 is valid at any time, by evaluating it, for example, at present time $t_{0}$ we can determine $k(\chi)$ as

$$
k(\chi)=H_{0}(\chi)^{2} A_{0}(\chi)^{2}\left(\Omega_{m 0}(\chi)+\Omega_{\Lambda 0}(\chi)-1\right)
$$

where $H_{0}(\chi) \equiv H\left(t_{0}, \chi\right)$ and $\Omega_{m 0}(\chi) \equiv \Omega_{m}\left(t_{0}, \chi\right)$ are the present day value of the (transverse) expansion rate

$$
H(t, \chi) \equiv \frac{\dot{A}}{A}
$$

and of the density parameter

$$
\Omega_{m}(t, \chi) \equiv \frac{8 \pi G}{3 H^{2}} \tilde{\rho}
$$

respectively. The curvature function $k(\chi)$ rests, in a degenerate way, on two unknown gauge functions $H_{0}(\chi)$ and $A_{0} \equiv A\left(t_{0}, \chi\right)$. We can conveniently remove this degeneracy by arbitrarily fixing the angular scale $A_{0}(\chi)$. This must be a smooth and invertible positive function, and in what follows we choose the conventional gauge of the literature and set $A_{0}(\chi)=\chi$. In other terms, we label each dust shell by means of its present day areal radius. Once a given matter density $\rho_{m}$ (or equivalently $\Omega_{m 0}(\chi)$ ) and a present day transverse expansion rate $H_{0}(\chi)$ are chosen, Eq. (7) can be solved to determine the remaining degree of freedom $A(t, \chi)$.

\section{REDSHIFT}

The redshift is defined in terms of the photon wave-vector as [56]

$$
1+z=\frac{\left(u_{\alpha} k^{\alpha}\right)_{e}}{\left(u_{\alpha} k^{\alpha}\right)_{0}}
$$

where the suffix $e$ and 0 indicate quantities that are computed at emission and present time respectively. The wave-vector components satisfy $k^{\alpha} k_{\alpha}=0$ and $k^{0}$, the physical energy of the photon, depends at most on $t$ and $\chi$ because of the rotational symmetries of the line element. We thus deduce that

$$
1+z=\frac{k^{0}(t, \chi)}{k^{0}\left(t_{0}, 0\right)}
$$

where we have set $\chi_{e}=\chi, \chi_{0}=0$ and $t_{e}=t$. The spatial and temporal evolution of the photon energy is accounted for by the geodesic equation for massless particles (3), which can be recast as the following partial differential equation

$$
\frac{\partial\left(\alpha k^{0}\right)}{\partial t}=\frac{\partial k^{0}}{\partial \chi} .
$$

By changing variable $U(t, \chi)=\alpha(t, \chi) k^{0}(t, \chi)$ and supposing that it exists a characteristic curve $t=t(\chi)$ on which the function $U(t(\chi), \chi)$ satisfy an ordinary differential equation, Eq. (14) becomes

$$
\frac{d U}{d \chi}-\frac{\alpha^{\prime}}{\alpha} U=\frac{\partial U}{\partial t}\left(\alpha+\frac{d t}{d \chi}\right)
$$

The equation is satisfied if

$$
\frac{d t}{d \chi}=-\alpha(t, \chi)
$$

which shows that the characteristic curve is indeed the null geodesic of the LTB metric, and

$$
\frac{d U}{d \chi}=\frac{\alpha^{\prime}}{\alpha} U
$$

It follows that the general solution, which depends on an arbitrary function $g$, fixed by the initial data, is

$$
U(t, \chi)=g\left(\chi+\int \frac{d t}{\alpha}\right) e^{\int \frac{\alpha^{\prime}}{\alpha} d \chi}
$$

and therefore the redshift is

$$
1+z=\frac{\alpha\left(t_{0}, 0\right)}{\alpha(t(\chi), \chi)} e^{\int_{0}^{\chi} \frac{\alpha^{\prime}}{\alpha} d \chi},
$$

where the integral is performed along the photon path $t=$ $t\left(\chi, t_{0}\right)$ solution of 16 .

The previous formula makes explicit the fact that the redshift depends not only on the time of emission and reception of the photon but also on the specific geodesic path of the photon $t=t(\chi)$. In the limiting case of a FRW model $(\alpha=a$ and $\alpha^{\prime}=0$ ) the redshift expression converges to the standard model one $1+z=a(t) / a\left(t_{0}\right)$, with the change in frequency resulting from the relative motion of freely falling matter. In the LTB model, instead, the varying gravitational potential between the observer and the emitter also contributes to the relative change in the photon frequency. As a result the redshift depends on the integrated variation of the gravitational field along the photon null geodesics. 
Expression (18 ) shows that, under particular circumstances, the redshift could be a non-monotonic function of the look-back time or of the radial coordinate $\chi$. Photons emitted at two different radial positions, or two different times, might be characterized by the same redshift. Indeed, the argument of the integral is not necessarily positive, and indeed it may change sign when the radial scale factor $\alpha$ is a non monotonic function of $\chi$ (while still being monotonic in the $t$ variable). This might happen even if the scale factor $A$ is a monotonic function of both temporal and radial coordinates. A distinctive consequence of this phenomenology will be presented in Sec. VI

For small physical separations $\delta \chi$ between the light source and the receiver, a series expansion to leading order of (18) results in a formula similar to the Hubble-Lemaitre expansion law, i.e.

$$
z \approx \frac{\dot{\alpha}_{0}}{\alpha_{0}} r
$$

where $r \equiv \alpha_{0} \delta \chi$. It is straightforward to show that $\dot{\alpha}_{0} / \alpha_{0}=$ $\dot{A}_{0} / A_{0}$, however for $t \neq t_{0}$ or $\chi \neq 0$ the ratio $\dot{\alpha} / \alpha$ does not coincide with $H=\dot{A} / A$, which encapsulates information on how different shells of matter moves under the action of gravity. To emphasise this we define the longitudinal Hubble function as

$$
H_{\|} \equiv \frac{\dot{\alpha}}{\alpha}
$$

Finally, by taking total derivatives of (18) with respect the $t$ and $\chi$, one recovers standard expressions quantifying how the redshift change when either the photon-flight time or the comoving coordinate interval between source and receiver vary. The equations that specify the past null cone are

$$
\begin{aligned}
& \frac{d z}{d t}=-(1+z) H_{\|} \\
& \frac{d z}{d \chi}=(1+z) \alpha H_{\|}
\end{aligned}
$$

respectively. These are the pair of coupled differential equations that determine the relations between the coordinates and the redshift, i.e. $t(z)$ and $r(z)$.

\section{REDSHIFT DRIFT}

Now consider a pair of photons that are both emitted and received by the same comoving objects at radial coordinates $\chi$ and $\chi_{0}=0$, respectively. Suppose that they are emitted(/received) at two instants $t$ and $t+\delta t\left(/ t_{0}\right.$ and $\left.t_{0}+\delta t_{0}\right)$, which are separated by a time interval over which cosmological changes cannot be anymore overlooked (typically on timescales of the order of a few years).

Formula 18 tells us that the redshift of a photon is formally a functional $z=z\left(t_{0}, t, \chi(t)\right)$ which depends on the specific geodesic path of the photon $\chi=\chi(t)$, on top of the integration boundaries $t$ and $t_{0}$. At the same coordinate time $t^{\prime}$, two different photons, emitted at two different times by the same comoving source, will be located at different radial coordinates $\chi^{\prime}$ and $\chi^{\prime}+\delta \chi$. If these two photons, emitted with a time delay $\delta t$ are detected by the comoving observer with a time lag $\delta t_{0}$, then the difference in their redshifts can be determined by computing the following variational quantity

$$
\delta z=z\left(t_{0}+\delta t_{0}, t+\delta t, \chi(t)+\delta \chi(t)\right)-z\left(t_{0}, t, \chi(t)\right) .
$$

or, in first order approximation, as

$$
\delta z=\frac{\partial z}{\partial t} \delta t+\frac{\partial z}{\partial t_{0}} \delta t_{0}+(1+z) \int_{t}^{t_{0}} d t^{\prime} \delta \chi\left(t^{\prime}\right) \frac{\delta z}{\delta \chi\left(t^{\prime}\right)}
$$

where $\delta z / \delta \chi\left(t^{\prime}\right)$ is the functional derivative of the redshift which, using 18 , evaluates to

$$
\frac{\delta z}{\delta \chi\left(t^{\prime}\right)}=(1+z) \partial_{\chi} H_{\|}\left(t^{\prime}, \chi\left(t^{\prime}\right)\right) .
$$

Since, by definition, time dilations are related as (see Appendix B for a detailed discussion)

$$
\frac{\delta t_{0}}{\delta t}=(1+z)
$$

by plugging the expressions 18 and 24 into Eq. 22 we obtain

$$
\begin{aligned}
\frac{\delta z}{\delta t_{0}}= & (1+z) \int_{t}^{t_{0}} \frac{\partial_{\chi} H_{\|}\left(t^{\prime}, \chi\left(t^{\prime}\right)\right) d t^{\prime}}{\alpha\left(t^{\prime}, \chi\left(t^{\prime}\right)\right)\left(1+z\left(t^{\prime}, \chi\left(t^{\prime}\right)\right)\right)} \\
& +H_{\|}\left(0, t_{0}\right)(1+z)-H_{\|}(\chi(z), t(z))
\end{aligned}
$$

where $t(z)$ and $\chi(z)$ are calculated along the ray path of the photon. As for the redshift [see Eq. 18], the redshift drift is also independent from the transverse scale factor $A$ and uniquely depends on the longitudinal Hubble function. Moreover, note that Eq. (25) reduces to the standard FRW formula when radial inhomogeneities vanish.

Up to this point, the discussion has relied solely on kinematic concepts, with no reference at all to the dynamical evolution of the metric. Indeed, Eq. 25 can be used to predict redshift drift in any spherically symmetrical spacetime regardless of whether its evolution satisfies Einstein's equations. However, if the metric changes in time according to the standard field equations of general relativity (see Sec. II B], the degrees of freedom of the LTB metric can be reduced. The price to pay is that the redshift drift becomes model dependent. The gain is that its redshift dependence can be easily predicted since the redshift drift becomes a function of the transverse expansion rate $H$. The model-dependent formula for the redshift drift can be obtained by simply replacing $\alpha=A^{\prime}(\chi)$ and

$$
H_{\|}=H\left(1+\frac{(\log H)^{\prime}}{(\log A)^{\prime}}\right) \text {. }
$$

in (25).

The cosmological relevance of the rate of change of the redshift drift itself, i.e. the next order time derivative of the redshift drift, has been pointed out by [57], and [44] have provided illustrations of the power of this observable in discriminating different cosmological models. These authors also emphasizes that such a measurement is well within the reach of 
the SKA Phase 2 array as well as of ELT-HIRES (albeit, in this case, with less sensitivity). A complementary goal of this article is therefore also to predict the amplitude and scaling of the second time derivative of the redshift within the framework of LTB cosmologies.

By proceeding as before, and taking the variation of the redshift drift formula, we can predict the amplitude and scaling of the second time derivative of the redshift within the framework of LTB cosmologies. The rate at which the drift changes is given by

$$
\begin{aligned}
\frac{\delta^{2} z}{\delta t_{0}^{2}}= & (1+z)\left[\frac{d H_{\|}}{d t_{0}}+g_{0}+\int_{t}^{t_{0}} \frac{\partial_{\chi} g}{\alpha(1+z)} d t^{\prime}\right]+ \\
& (1+z)^{-1}\left[\left(\frac{\delta z}{\delta t_{0}}\right)^{2}-H_{\|}\left(H_{\| 0}+\frac{\delta z}{\delta t_{0}}\right)+\frac{d H_{\|}}{d t}\right]-H_{\| 0} H_{\|}
\end{aligned}
$$

where

$$
\begin{gathered}
g\left(t_{0}, t, \chi(t)\right)=\frac{\partial_{\chi} H_{\|}(t, \chi(t)) d t}{\alpha(t, \chi(t))\left(1+z\left(t_{0}, t, \chi(t)\right)\right)} \\
\frac{d H_{\|}}{d t}=\frac{\partial H_{\|}}{\partial t}+\frac{\partial H_{\|}}{\partial \chi} \frac{d \chi}{d t_{0}}=\dot{H}_{\|}-\frac{H_{\|}^{\prime}}{\alpha}
\end{gathered}
$$

and where a suffix 0 means that the quantities are evaluated at $\chi=0$ at present time $t_{0}$.

Again, in the limit in which the relevant quantities do not depend on the radial coordinate $\chi$, the standard FRW formula

$$
\frac{\delta^{2} z}{\delta t_{0}^{2}}=\dot{H}_{0}(1+z)+H_{0}^{2}(1+z)-H_{0} H-\frac{\dot{H}}{1+z}
$$

is recovered.

\section{SYSTEMATIC EFFECTS}

Here we investigate potential systematics that might perturb the redshift drift measurement. On top of numerical artifacts that arise because of the different approximation schemes with which the redshift drift is defined and computed, we also analyse the impact of physical effects, such as proper motions of the sources or the observer.

\section{A. Redshift drift definition}

There are three possible way in which the redshift drift can be defined. The definition that most closely capture the essence of the phenomenon is IV] where the redshift is computed using the formula (18) [and the time dilation using Eq. [24]. No approximations are involved in this definition that we refer to as method 1 .

A second way of defining the redshift drift is via the linear approximation Eq. (22) applied to the integral solution 18 . This approach, here called method 2, captures leading order contributions to the time change of the redshift, and leads to the explicit formula 25. Conceptually, this approach differs from the approximation scheme, applied almost ubiquitously in literature, which consists in linearizing the geodesic equations [cf. eqs. 20, and 21]] and computing, numerically, the redshift drift. According to this last strategy (method 3), the amplitude of the drift follows from solving the approximated differential equation

$$
\frac{d}{d \lambda} \delta z(\lambda)=\frac{\partial \chi}{\partial \lambda} \dot{\alpha} \delta z+(1+z) \ddot{\alpha} \delta z(\lambda)
$$

with the initial condition $\delta z(0)=0$ along the geodesic path of the photon $\chi(\lambda), t(\lambda)$ (see Appendix $\mathrm{C}$ ).

Given the explicit solution for the redshift in LTB cosmologies given in Eq. (18), we are in measure to compare all these definitions, check their mutual consistency and thus the effectiveness of the approximations involved. The results of applying these schemes to the ALTB model is shown in Fig. 1 .

Errors due to the linear approximation involved in both methods 2 and 3 as well as the numerical imprecision in computing the geodesics path of the photons (via equations 20, and (21)) are negligible, with the relative imprecision of both methods 2 and 3 with respect to method 1 of order $\sim 5 \times 10^{-5}$ over all the redshift range explored. The "theoretical" noise is statistically insignificant, if compared to the "observational" imprecisions expected for the measurements of the redshift drift signal, and there is no need to devise a more refined analytical way for computing the redshift drift nor a more stable numerical way to solve for the geodesics in LTB cosmologies.

The relative imprecision in computing the redshift drift according to methods 2 and 3 is even smaller, of order $\sim 10^{-7}$. This insignificant residual discrepancy results from the linearisation procedure being applied to the differential equations before integration (method 3) or directly to the integral expression for the redshift (method 2).

As was originally noted by [52], the analytic expression for the redshift drift obtained by [4] (see their Eq. 9) appears to differ from the numerical results predicted by method 3 , although both computational schemes correctly reproduce the standard model predictions in the FRW limit. This discrepancy is explicitly evident if we compare our analytical expression [cf. Eq. [25]] with the redshift drift expression given in Eq. 2.28 of [58]. We illustrate the amplitude of the mismatch for two different LTB models, the mLTB and the $\Lambda$ LTB cosmologies (described in VI) in Fig. 2. While [52] suggests that the disagreement might be caused by a different definition of the redshift drift observable, this in reality results from [4] estimating the redshift drift a simple differential, instead, as we did, as functional derivative.

\section{B. Local peculiar velocity drift}

The dominant correction to the redshift drift signal arises from the time change of the peculiar velocity of galaxies in the time lag $\delta t_{0}$ between two redshift measurements. Since the peculiar velocities of the various cosmic sources are, on large 


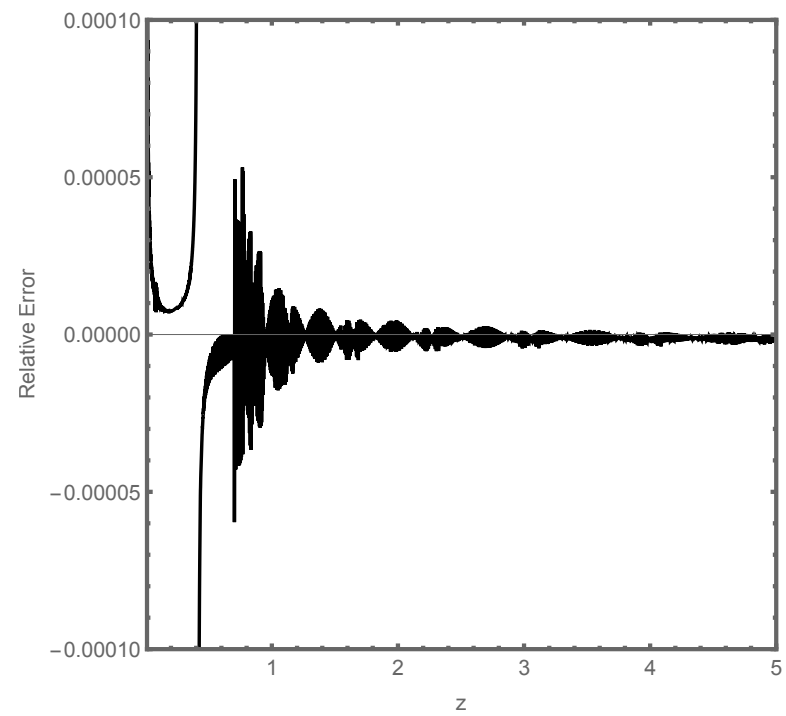

FIG. 1: Relative error in the redshift drift estimated according to models 1 and 2. The relative error is calculated as (model 1 - model $2) /($ model 2$)$. We have assumed the $\Lambda$ LTB universe as reference cosmology (see Sec. VI).

cosmic scales, uncorrelated, they do not contribute to the signal if not in increasing its variance. Indeed, it was shown by [59], using linear perturbation theory of the standard model of cosmology, that stochastic noise does not to contribute at a level greater than $0.1 \%$ for $z \leq 5$. However [60] showed that, on the characteristic time scale of the redshift drift $\left(H_{0}\right)$, the peculiar velocity of the observer (the Local Group center) change due to the competing effect of global cosmic expansion and local gravitational pull of surrounding cosmic mass structures. We here quantify whether the systematic drift of the observer's velocity, computed in a LTB spacetime, has any measurable effect on the redshift drift.

If the observer moves $\left(U^{i}\left(t_{0}, \chi_{0}\right) \neq 0\right)$, then the observed redshift $z^{o}$ of a comoving source is related to the cosmological redshift $z$ as [cf Eq. [12] ]

$$
1+z^{o}=\frac{1+z}{1+\boldsymbol{\beta}^{o} \cdot \boldsymbol{n}}
$$

where $\beta^{i}=\sqrt{\left|g_{i i}\right|} U^{i}$ is the physical (peculiar) velocity and $\boldsymbol{n}$ is the line of sight direction. At leading order, and setting $\beta=\alpha d \chi / d t$,

$$
\frac{\delta z}{\delta t}=\frac{\delta z}{\delta t_{0}}-\dot{\beta}
$$

since the photons propagates along the radial coordinate $\chi$ for an observer sitting at the center of symmetry of the LTB metric (we neglect higher order terms arising from the fact that the observer will move out of the central position due to its peculiar velocity.) We can estimate the additional dipole modulation of the redshift drift signal by computing the radial component of the acceleration of the central observer in a LTB
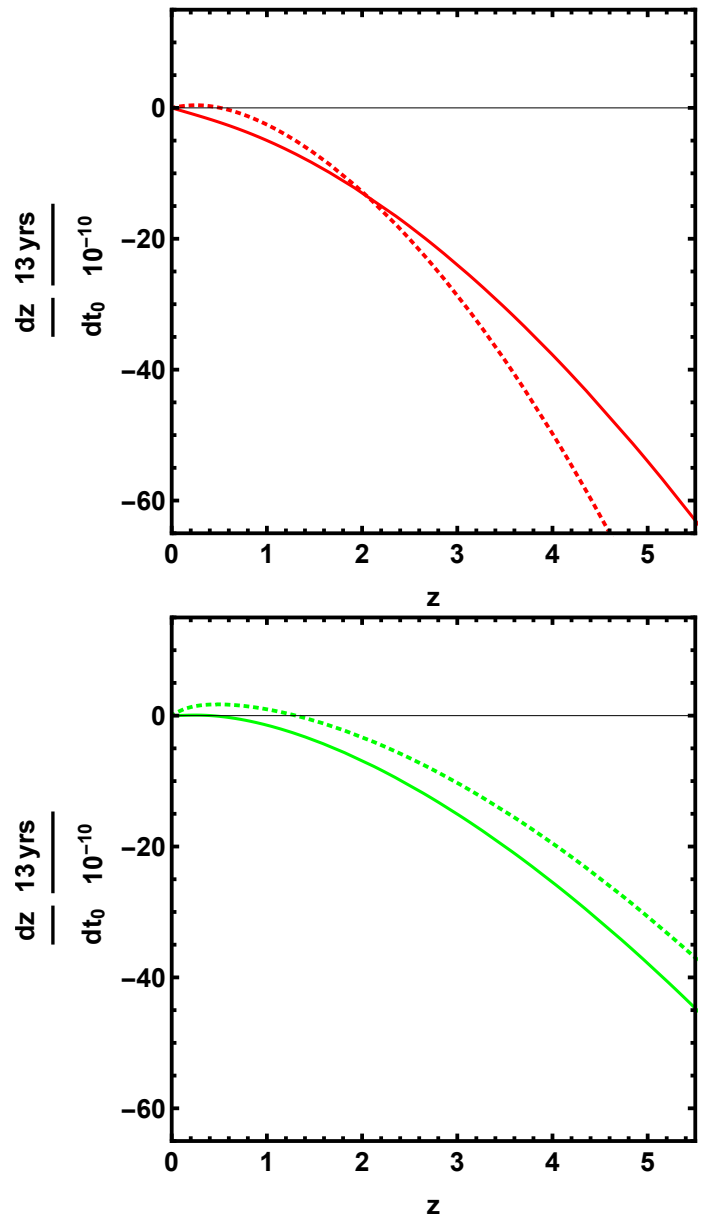

FIG. 2: Redshift drift estimated using Eq. 25 (solid line) and using Eq. 2.28 of [58] (dashed line) for two different LTB scenarios: the mLTB model (left panel) and the $\Lambda$ LTB model (right panel) (see VI).

metric. From Eqs. (3) and (4) we obtain that

$$
\dot{\beta}=-H_{\|} \beta\left(1-\beta^{2}\right)-\frac{\alpha^{\prime}}{\alpha^{2}} \beta^{2} .
$$

If the LTB metric evolves according to the EFE then $\alpha^{\prime}=0$ so that the acceleration vector is proportional to the velocity one. At leading order, we thus conclude that

$$
\dot{\beta}^{o} \approx H_{0} \beta^{o}\left(1+\left.\frac{(\log H)^{\prime}}{(\log A)^{\prime}}\right|_{0}\right) .
$$

For relevant models (in which the difference between the longitudinal and the transverse Hubble function is not greater than $10 \%$ as for example the models discussed in Sec. VI. and in which the central observer velocity is not greater than $\sim 700 \mathrm{~km} / \mathrm{s}$, the standard model velocity of the Local Group of galaxies with respect to the cosmic microwave background (CMB), this systematic effect is not expected to exceed $1 \%$ in the direction of motion of the observer. It is interesting to note that even if future redshift drift data were to reach a comparable level of accuracy, the dipolar character of the contaminating signal would facilitate its identification and subtraction. 

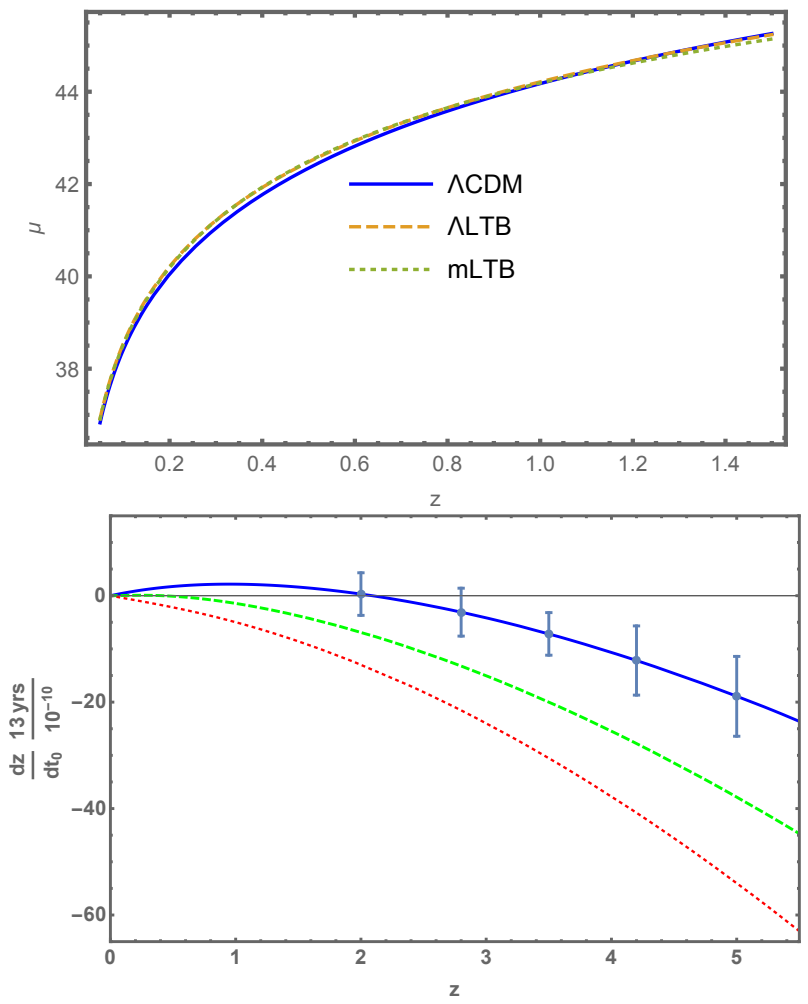

FIG. 3: Upper: distance modulus calculated in $\Lambda \mathrm{CDM}$ (solid line), $\Lambda$ LTB (dashed line) and in $m$ LTB (dotted line) respectively. Lower: redshift drift expected in the models presented in the upper panel. The $1 \sigma$ error bars and the data range are those predicted for CODEX observations over a 13-year time span. Data points and error are taken from [54].

\section{APPLICATION WITH EXPLICIT LTB MODELS}

In this section, we review some popular LTB models published in the literature that claim to reproduce the predictions of the $\Lambda \mathrm{CDM}$ model. We premise that we are not interested in their physical feasibility or cosmological soundness, but in their practical utility as toy models in order to highlight the potential of the redshift drift observable in resolving some of the degeneracies that plague LTB cosmologies when compared to observational data.

The first set of models are those that were shown by [20] to reproduce the Hubble diagram of supernovae. The first model (we call it mLTB) describes a LTB universe which is comprised only of matter $\left(\Omega_{m 0}(\chi)=1\right)$, while the second one ( $\Lambda \mathrm{LTB})$ incorporates the contribution of the cosmological constant $\Lambda$ and satisfies to the flatness constraint $\Omega_{m}(\chi)+\Omega_{\Lambda}(\chi)=1$. The rationale for choosing them is that the spacetime evolution of the scale factor $A(t, \chi)$ can be computed analytically, making the results more transparent. By integrating Eq. (7) for $\Omega_{m}+\Omega_{\Lambda}=1$ we obtain the following
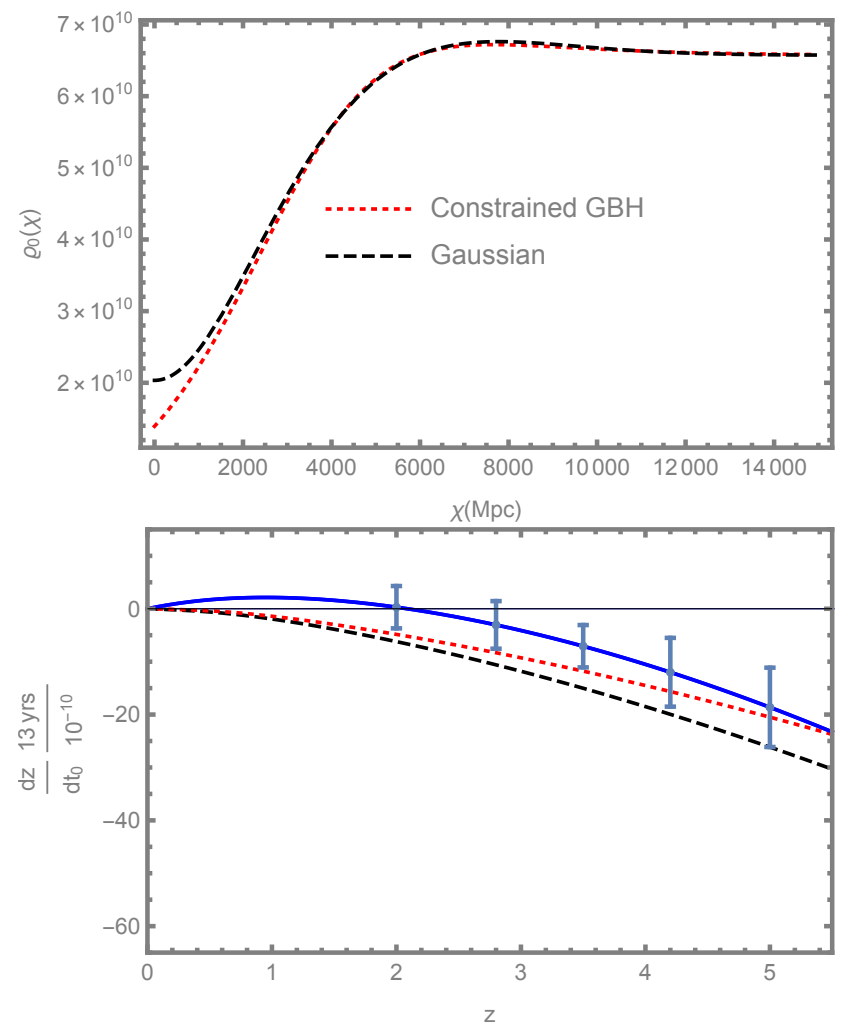

FIG. 4: Upper: the spatial scaling of the present day physical matter density profile $\rho_{0}(\chi)$ (in units $M_{\odot} M p c^{-3}$ ) for the constrained GBH (red dotted line) and the Gaussian (black dashed line) void models. Lower: comparison of the redshift drift expected in the standard $\Lambda \mathrm{CDM}$ scenario (blue solid line) and in the above LTB models which best fit SNIa, BAO and CMB data. Data points and $1 \sigma$ error are the same as in Fig. 3.

expression for the scale factor:

$$
\begin{aligned}
\frac{A}{A_{0}} & =\left[\cosh \left(\frac{3}{2} \sqrt{1-\Omega_{m, 0}} H_{0}\left(t-t_{0}\right)\right)\right. \\
& \left.+\frac{1}{\sqrt{1-\Omega_{m, 0}}} \sinh \left(\frac{3}{2} \sqrt{1-\Omega_{m, 0}} H_{0}\left(t-t_{0}\right)\right)\right]^{2 / 3}
\end{aligned}
$$

which reduces to $A / A_{0}=\left[1+3 / 2 H_{0}(\chi)\left(t-t_{0}\right)\right]^{2 / 3}$ in the case of the mLTB model.

Both models are described by the same present day transverse expansion rate profile

$$
H_{0}(\chi)=H_{0}(\infty)+\Delta H e^{-\chi / \chi_{0}}
$$

with parameters fine tuned to fit the Hubble diagram of SNIa data (see Fig. 3). Specifically, the mLTB model is characterised by $H(\infty)=48.7 \mathrm{~km} / \mathrm{s} / \mathrm{Mpc}, \Delta H=16.8 \mathrm{~km} / \mathrm{s} / \mathrm{Mpc}$ and $\chi_{0}=1400 \mathrm{Mpc}$, while the $\Lambda$ LTB scenario has best fitting parameters $H_{0}(\infty)=58 \mathrm{~km} / \mathrm{s} / \mathrm{Mpc}, \Delta H=8 \mathrm{~km} / \mathrm{s} / \mathrm{Mpc}$, $\chi_{0}=600 \mathrm{Mpc}$ and $\Omega_{\Lambda 0}(0)=0.33$.

The second set includes more sophisticated models that are tuned to agree with multiple observational probes, not just the Hubble diagram of SNIa. They are also meant to satisfy the- 
oretical stability constraints. For example the simple inhomogeneous models mLTB and $\Lambda$ LTB share the assumption that the the universe has an inhomogeneous big bang, i.e. it came into being at different times at different positions $\chi$. However, variations in the cosmic age function can be related to the decaying modes in the theory of linear perturbations [61] which, in turn, would imply the existence of large fluctuations at remote epochs, in contradiction with the remarkable homogeneity of the CMB spectrum. On the contrary, it is much more constructive, from a physical point of view, to assume that spherical inhomogeneities develop and grow over time, due to gravitational instabilities, in an otherwise uniform universe. Therefore, we would like to consider scenarios in which a spherically symmetric inhomogeneity is asymptotically embedded in the uniform FRW spacetime, i.e. converge to the standard metric of the universe at large distances and at early times. This is achieved by considering models in which the present day reduced matter density parameter is

$$
\Omega_{m 0}(\chi)=\Omega_{m 0}^{s}\left(1+\delta_{\Omega}\right)
$$

where $\Omega_{m 0}^{s}$ is the reduced density of the embedding standard flat $\Lambda C D M$ model. We thus extend the set to include the constrained model of [21] (hereafter constrained GBH)

$$
\delta_{\Omega}=\delta_{v} \frac{1-\tanh \left(\frac{\chi-\chi_{s}}{2 \Delta \chi_{s}}\right)}{1+\tanh \left(\frac{\chi_{s}}{2 \Delta \chi_{s}}\right)}
$$

where $\delta_{v}$ is the relative difference between the reduced density parameter at the center of the void and the asymptotic FRW value $\chi_{s}$ is the typical size of the void, and the parameter $\Delta \chi_{s}$ controls the steepness of the transition between the interior and exterior of the underdensity. When implemented with carefully tuned parameters, this model is in agreement with the Hubble diagram of SNIa, the Baryon Acoustic Oscillation (BAO), the CMB and also the age of old high redshift objects [62]. For the purposes of the present analysis, we adopt the best parameters fitted by [63] $\left(\Omega_{m 0}^{s}=1, \delta_{v}=-0.88, \chi_{s}=3.72\right.$ Gpc, $\left.\Delta \chi_{s}=1.68 \mathrm{Gpc}\right)$.

Additionally, we also also consider the Gaussian void model [64],

$$
\delta_{\Omega}=\delta_{v} e^{-\left(\frac{\chi}{\chi_{s}}\right)^{2}}
$$

where the parameters have the same physical interpretation as in the previous case. We implement this void profile by setting $\Omega_{m 0}^{s}=1, \delta_{v}=-0.81, \chi_{s}=5.04 \mathrm{Gpc}$, which, according to [63], provide an equally satisfactory fit to the SNIa, BAO and CMB data. For both the constrained GBH and the Gaussian LTB models the spatial hypersurface at the big bang does not depend on the radial coordinate $\chi$, meaning that the radial scaling of the present day transverse expansion rate is fixed by the relation

$$
H_{0}(\chi)=\frac{H_{0}}{1-\Omega_{m 0}}\left[1-\frac{\Omega_{m 0}}{\sqrt{1-\Omega_{m 0}}} \sinh ^{-1} \sqrt{\frac{1-\Omega_{m 0}}{\Omega_{m 0}}}\right]
$$

where $H_{0}$ is chose so that the age of the universe is 13.8 Gyrs. For both these matter+curvature models the time and spatial
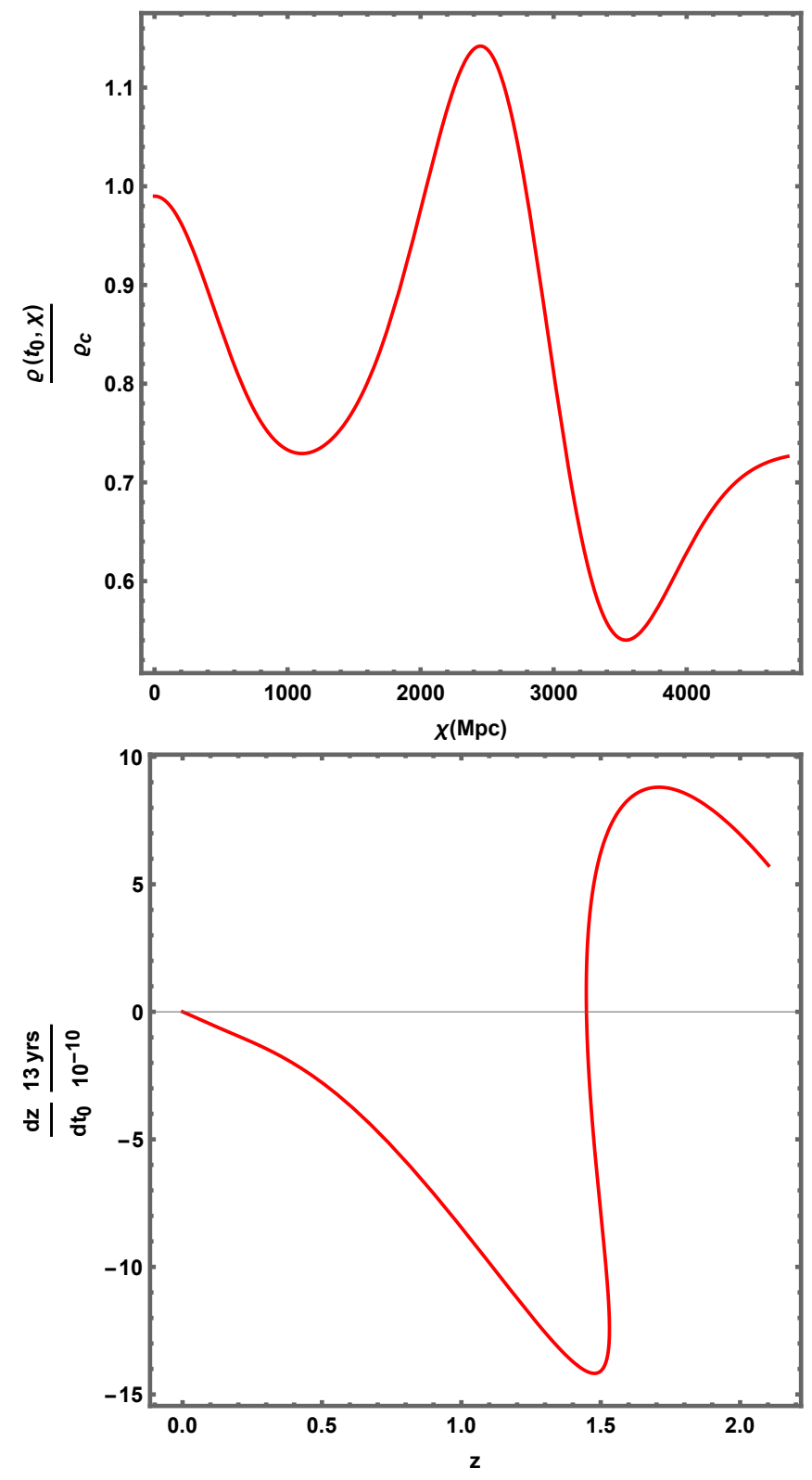

FIG. 5: Redshift drift in a cosmological model where the redshift is a non monotonic function of the radial coordinate $\chi$. Upper: matter density profile. Lower: redshift drift.

evolution of the transverse scale factor is given by the parametric equations

$$
\begin{aligned}
A(\eta, \chi) & =\chi \frac{\Omega_{m 0}}{1-\Omega_{m 0}}(\cosh \eta-1) \\
t(\eta, \chi) & =\frac{1}{H_{0}(\chi)} \frac{\Omega_{m 0}}{2\left(1-\Omega_{m 0}\right)^{3 / 2}}(\sinh \eta-\eta) .
\end{aligned}
$$

The resulting void density profiles, obtained by using eqs. 8 and 10 , are shown in the upper panel of Fig. 4 

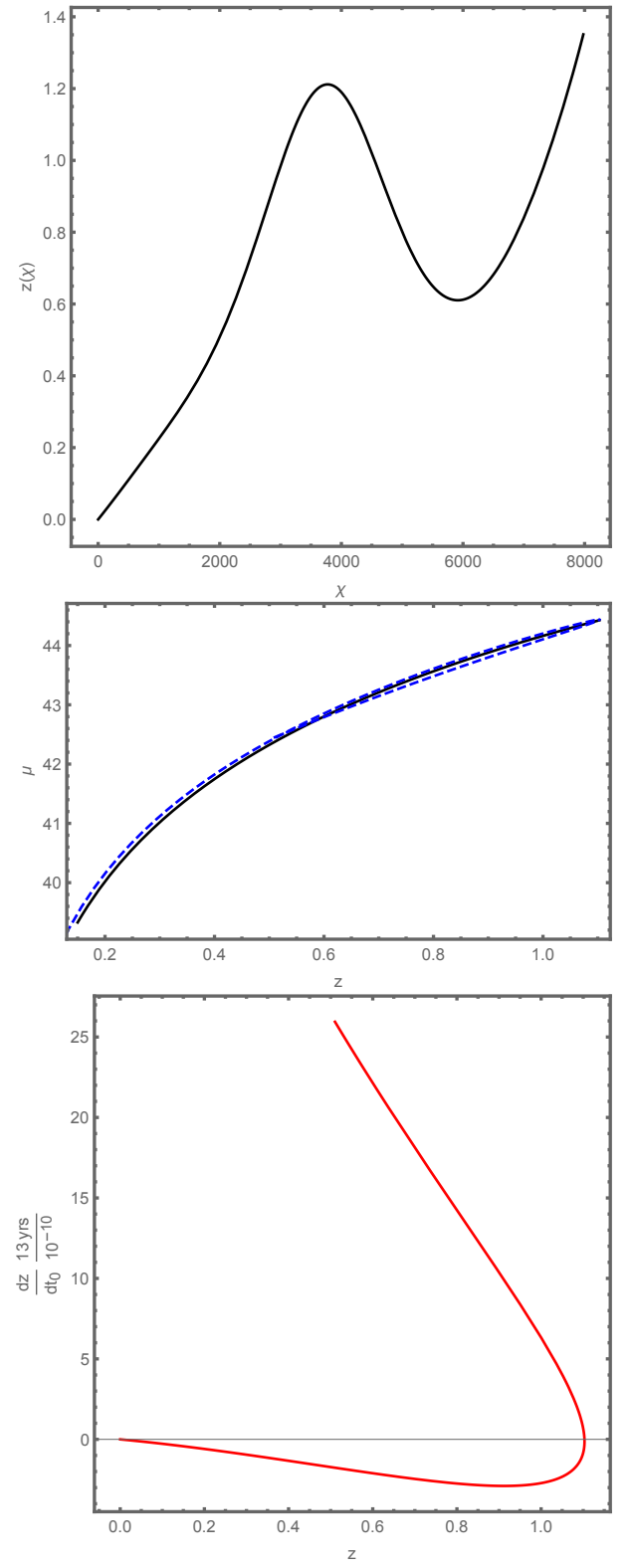

FIG. 6: Upper panel: redshift $z$ as a function of the radial coordinate $\chi$ along the null geodesic of a photon in a LTB model in which $\chi(z)$ is not a single valued function. Central panel: the Hubble diagram is plotted as a function of redshift for the LTB model shown above. In this particular model, photons emitted at two different epochs have the same redshift and the same transverse scale factor $A(t, \chi(t))$ and thus their Hubble diagram is degenerate and indistinguishable from that of the standard $\Lambda \mathrm{CDM}$ model. Bottom panel: redshift drift as a function of redshift for the same LTB model shown in the upper panel.

\section{A. Discussion}

The luminosity distance of an object at redshift $z$ is given by (see for example [65])

$$
d_{L}(z)=(1+z)^{2} A(\chi(z), t(z))
$$

where $\chi(z)$ and $t(z)$ are computed along the null geodesics of the photon using equations (20) and (21). The resulting distance modulus $\mu$ as a function of the redshift for the $\Lambda \mathrm{CDM}$ model and the mLTB and LTB models is shown in the upper panel of Fig. 3. By construction they do not differ appreciably. Indeed, because of the additional functional degrees of freedom induced by the spatial dependence of the transverse expansion rate, the Hubble diagrams of many physically distinctive inhomogeneous models are degenerate. This is mostly the consequence of this observable being an integral quantity of the redshift. If instead of the distance to cosmic objects we consider the time change of their redshift, we can restore some degree of predictability. This is shown on the bottom panel of Fig. 3 where the redshift drift for these very same three cosmological models is plotted as a function of redshift. LTB models whose magnitude-redshift predictions are degenerate with the standard Hubble diagram can be easily distinguished because they predict very different behaviors for the redshift drift. By inspecting Fig. 3 one can also appreciate how the data expected from future observational projects (the CODEX survey in this case) have the power necessary to discriminate not only the standard model from more exotic LTB-type scenarios, but also between various LTB models that would otherwise be indistinguishable on the basis of their Hubble diagram.

There are also LTB models that are compatible with a wider range of data, particularly the $\mathrm{BAO}$ and $\mathrm{CMB}$ measurements, which, in addition to predicting the same luminosity distance, are also characterized by void density profiles that are observationally indistinguishable. This is the case, for example, of the constrained GBH model and the Gaussian model shown in the top panel of Fig. 4 Interestingly, also these models predict redshift drift signals that are significantly different not only from the expectations of the standard $\Lambda \mathrm{CDM}$ model, but also from each other.

One can better appreciate the specificity of this phenomenon by contrasting it to what happens in the standard model. Even in a homogeneous and isotropic spatial background, two different models of dark energy (one for example in which the acceleration of the scale factor of the metric is contributed by the cosmological constant and another in which it originates from the 'negative' pressure exerted by a scalar field) can be fine-tuned to reproduce the same Hubble diagram of SNIa (see for example [66]). However, the redshift drift cannot disentangle these two different physical models since the predicted amplitude will also be degenerate.

The ability of the redshift drift probe in discriminating different inhomogeneous LTB models relies entirely on two essential features. First, although LTB models have a large number of functional degrees of freedom, at most only a subset of structural functions characterizing different void models can be indistinguishable. In particular, the various models considered in our analysis all exhibit the same spatial and temporal dependence of the transverse expansion rate $H$, although the scaling of the longitudinal expansion rate $H_{\|}$turns out to be very different and model dependent. For example, the maximum amplitude of the ratio $H / H_{\|}$, which occurs at about $\chi \sim \chi_{s}$, is significantly larger $(\sim 20 \%)$ in the constrained GBH 
model than in the Gaussian model. Consequently, observables such as redshift drift, which are sensitive to multiple structural parameters (in particular to $H_{\|}(t, \chi), H(0, t)$, and $\alpha(t, \chi)$ as can be seen in Eq. 25) and not only to the transverse expansion rate, as in the case of the luminosity distance, can be effectively used to singularize LTB models. In this sense, the Hubble diagram, like any observable sensitive to a limited set of LTB structural functions, is a poor diagnostic tool.

In addition, the functional nature of the redshift drift in spherically symmetric spaces is a key feature that facilitates model discrimination. This observable does not depend only on the redshift of the emitting source, as in the standard model of cosmology, but also on the geodesics taken by the photons to reach the observer. The path integral that goes into its definition (cf. 25) makes the redshift drift in spherically symmetric spaces a nonlocal and explicitly model-dependent quantity. For example, fine tuning the scaling of $H$ in different LTB models to reproduce the luminosity distance comes at the price of making the geodesic path of photon to vary from model to model. Observable as the redshift drift, being sensitive to the entire geodetic history of the signal, are therefore optimal tools for distinguishing various inhomogeneous patterns.

The non-local nature of the redshift drift in LTB cosmologies is responsible of some interesting phenomenology. The behaviour of the redshift drift at low $z$ follows from taking the lowest order terms of the series expansion of Eq. (25) around $t=t_{0}$

$$
\frac{\delta z}{\delta t_{0}} \approx-\left.\frac{H_{\|}^{\prime}}{\dot{\alpha}}\right|_{0} \delta t_{0}+H_{\|}\left(0, t_{0}\right) z-\dot{H}_{\|}\left(0, t_{0}\right) \delta t_{0}-H^{\prime}\left(0, t_{0}\right) \delta \chi
$$

which reduces to

$$
\frac{\delta z}{\delta t_{0}} \approx \frac{\ddot{\alpha_{0}}}{\dot{\alpha}_{0}} z=-\frac{1}{2} H_{0}(0) z\left(\Omega_{m 0}(0)-2 \Omega_{\Lambda_{0}}(0)\right) .
$$

According to the above equation, the redshift of a low- $z$ source decreases in time in a purely matter-dominated LTB model. In other terms, if dust is the only source of gravity, the redshift drift cannot be positive for objects close to the center of symmetry. In this respect, the matter-dominated LTB models behave as a homogeneous and isotropic universe filled with dust. However, contrary to standard model results, the redshift drift might eventually becomes positive at higher redshifts. This effect is shown in Fig. 5 together with the peculiar radial matter density profile that generates it. The phenomenon is induced by the non-monotonic scaling of $\alpha(\chi)$ which causes the functions $H_{\|}$and $\partial_{\chi} H_{\|}$to change sign along the photon geodesc. In this specific case $H_{\|}$becomes progressively more negative as the radial coordinate of the source increase, forcing the sign of the redshift drift to turn positive (see Eq. 25).

Other unconventional effects characterises the redshift drift in LTB cosmologies. One can engineer the shape of $H_{0}(\chi)$ such that redshift itself is no longer a monotonic function of the spacetime coordinates $t$ and $\chi$, or, which is equivalent, the time and radial coordinates are no longer single valued functions of the redshift along the null-geodesic path. One can exploit this functional degree of freedom associated to $H_{0}(\chi)$, for example, to design models where the redshift of very distant objects turn negative. More intriguingly, one can also devise physical models, i.e. LTB models with a strictly non negative matter density function $\rho_{m}$, where objects at different radial coordinates $\chi$ (thus emitting the photons at different epochs) have the same redshift and, thus, the same radial scale factor $A(t(z), \chi(z)$, or, equivalently, the same distance modulus $\mu(z)$. This peculiar phenomenon is shown in Fig. 6, where the Hubble diagram is plotted as a function of redshift. As the photons coming from these sources are not following the same geodesics path, the Hubble diagram degeneracy is broken when the redshift drift is analysed. Indeed, since the redshift drift is a non-local, path dependent function, two different values of the redshift drift can correspond to the same redshift value. In this context, means that the density matter is strictly non negative function.

\section{CONCLUSION}

Providing evidences of cosmological changes on human time-scales [60, 67, 68] is challenging. While the physics is transparent and fascinating, the signal is characteristically weak. Nevertheless, measurements of the redshift drift are certainly within the reach of large telescopes in the next 20 years [69]. It is thus worthwhile exploring the possibilities for using this observable to understand the cosmological impact of inhomogeneities in the large-scale structure of the universe.

In this context, our goal was to provide an analytical formula for calculating the redshift drift in spherically symmetric spaces. As a result, we have highlighted the functional character of this probe, a quantity that depends not only on the time instants at which photons from a source are emitted and received, but also on the null-geodesics traveled by the photons to reach the observer.

The non-local nature of the redshift drift in spherically symmetric spaces is at the origin of some peculiar phenomena. Arguably, the most interesting is the possibility of disentangling inhomogeneous models predicting the same Hubble diagram. Since the LTB spacetime has fewer symmetries than the standard metric of the universe, the distance-redshift relation of the standard $\Lambda C D M$ model can be reproduced with arbitrary precision using, for example, the freedom in the specification of the transverse expansion rate function $H_{0}(\chi)$. Even more maliciously, various inhomogeneous models, characterized by widely different structural parameters, predict distance moduli so similar that they resist the resolving power of data.

We have shown that the redshift drift has the potential to break this degeneracies and restore some predictive power to LTB models. This is important if we consider that the linear perturbation theory of LTB cosmological models, because of the technicalities it implies, is still in its infancy and that observable features of the large-scale structure of the universe, such as peculiar velocities, growth rates or power spectra matter density fluctuations etc., cannot be effectively used to resolve degeneracies appearing in the background sector of the theory.

One possible way to progress further and complete the 
study would be to evaluate the consequences of moving the observer's position away from the center of symmetry and quantify the dependence of the redshift drift on the specific off-centric location. We plan to expand on these issues in a forthcoming paper.

Acknowledgements. We would like to thank Julien Bel and Federico Piazza for useful discussions. This work was partially supported by the Institut Physique de l'Univers (IPHU Grant No. 013/2020) and by the Programme National GRAM of CNRS/INSU with INP and IN2P3 co-funded by CNES.

\section{Appendix A: Evolution of the scale factors of the LTB metric}

The Einstein field equations for a LTB spacetime containing a non-relativistic perfect fluid, i.e. dust with an equation of state $(p=0)$, and a non-dilutive dark energy component $\rho_{\Lambda}=$ const are

$$
\frac{1}{A^{2}}+\left(\frac{\dot{A}}{A}\right)^{2}+2 \frac{A^{\prime} \alpha^{\prime}}{A \alpha^{3}}-2 \frac{A^{\prime \prime}}{A \alpha^{2}}+2 \frac{\dot{\alpha} \dot{A}}{A \alpha}-\left(\frac{A^{\prime}}{A \alpha}\right)^{2}=8 \pi G\left(\rho_{m}+\rho_{\Lambda}\right)
$$

$$
\dot{A}^{\prime}=A^{\prime} \frac{\dot{\alpha}}{\alpha}
$$

$$
\begin{aligned}
& \frac{1}{A^{2}}+2 \frac{\ddot{A}}{A}+\left(\frac{\dot{A}}{A}\right)^{2}-\left(\frac{A^{\prime}}{A \alpha}\right)^{2}=8 \pi G \rho_{\Lambda} \\
& \frac{\ddot{A}}{A}-\frac{A^{\prime \prime}}{A \alpha^{2}}+\frac{\dot{A} \dot{\alpha}}{A \alpha}+\frac{A^{\prime} \alpha^{\prime}}{A \alpha^{3}}+\frac{\ddot{\alpha}}{\alpha}=8 \pi G \rho_{\Lambda} .
\end{aligned}
$$

Eq. A2 can be integrated with respect to $t$ resulting in

$$
\alpha(\chi, t)=C(\chi) A^{\prime}(\chi, t)
$$

where $C$ is a function of $\chi$ only which, being arbitrary, we redefine as $C(\chi)=1 / \sqrt{1-k(\chi)}$. Once Eq. A5 is inserted in (A1), (A3) and Eq. (A4), the system reduces to the two independent equations

$$
\frac{\dot{A}^{2}+k}{A^{2}}+\frac{2 \dot{A} \dot{A}^{\prime}+k^{\prime}}{A A^{\prime}}=8 \pi G\left(\rho_{m}+\rho_{\Lambda}\right)
$$

and

$$
\frac{\dot{A}^{2}+2 A \ddot{A}+k}{A^{2}}=8 \pi G \rho_{\Lambda} .
$$

We can further simplify by multiplying Eq. A7 by $A^{2} \dot{A}$ and integrating it over time. We find

$$
A \dot{A}^{2}=\frac{8 \pi G}{3} \rho_{\Lambda} A^{3}-k A+\frac{8 \pi G}{3} A^{3} \tilde{\rho}
$$

and, thus,

$$
\left(\frac{\dot{A}}{A}\right)^{2}+\frac{k}{A^{2}}=\frac{8 \pi G}{3}\left(\tilde{\rho}+\rho_{\Lambda}\right)
$$

where $\tilde{\rho}(t, \chi)$ is an arbitrary integration function such that the product $A^{3} \tilde{\rho}$ depends only on the radial coordinate $\chi$. Its relation to the physical matter density $\rho_{m}$, the quantity that appears in the stress energy tensor, is obtained as follows. First we derive A8 with respect to the radial coordinate and replace the result into Eq. A6 to obtain

$$
\left(A^{3} \tilde{\rho}\right)^{\prime}=3 \rho_{m} A^{2} A^{\prime} .
$$

which, upon integration, gives

$$
\tilde{\rho}(t, \chi)=3 \frac{\int_{0}^{\chi} \rho_{m} A^{2} A^{\prime} d \chi}{A^{3}}
$$

where we used the fact that $\tilde{\rho}(0, t) A^{3}(0, t)=0$. The inverse relationship can be recovered by performing the differentiation in $\mathrm{A} 10$

$$
\rho_{m}=\tilde{\rho}+\tilde{\rho}^{\prime} \frac{A}{3 A^{\prime}} .
$$

The flat average density of matter $\tilde{\rho}$ allows us to rewrite the equations of motion of the scale factor $A(t, \chi)$ of the LTB metric in a way similar to those governing the evolution of the scale factor $a(t)$ of the FRW metric in the standard model of cosmology, i.e. in a Friedmann-like form. Indeed, by deriving with respect to time Eq. A9] as well as the time-independent product $A^{3} \tilde{\rho}$ we get

$$
\frac{\ddot{A}}{A}=-\frac{4 \pi G}{3}\left(\tilde{\rho}-2 \rho_{\Lambda}\right)
$$

and

$$
\dot{\tilde{\rho}}+3 \frac{\dot{A}}{A} \tilde{\rho}=0
$$

i.e. the acceleration and continuity equations respectively.

The acceleration of the longitudinal scale factor is

$$
\begin{aligned}
\frac{\ddot{\alpha}}{\alpha}=\frac{\ddot{A}^{\prime}}{A^{\prime}} & =-\frac{4}{3} \pi G\left[\frac{(\tilde{\rho} A)^{\prime}}{A^{\prime}}-2 \rho_{\Lambda}\right] \\
& =\frac{\ddot{A}}{A}-\frac{4}{3} \pi G\left[\frac{(\tilde{\rho} A)^{\prime}}{A^{\prime}}-\tilde{\rho}\right]
\end{aligned}
$$

which, in the FRW limit (no radial inhomogeneities), gives $\ddot{\alpha} / \alpha=\ddot{A} / A$.

The longitudinal and transverse expansion rates are related as

$$
H_{\|}=H+\frac{A}{A^{\prime}} H^{\prime}=H+\frac{A^{2}}{2 \dot{A} A^{\prime}}\left[\frac{8 \pi G}{3} \tilde{\rho}^{\prime}-\left(\frac{k}{A^{2}}\right)^{\prime}\right]
$$

and, at the center of symmetry $(\chi=0)$, they coincide $H_{\|}(0, t)=H(0, t)$. 


\section{Appendix B: Time dilation formula in a LTB cosmology}

Although the time dilation formula (24) can be taken as the very definition of what redshift is, i.e. the relative change in the proper frequency of a signal as measured at emission and at reception [65], it is instructive to see how this result can be explicitly derived in the framework of the LTB spacetime. In doing so, we will have the opportunity to restate the formula we obtained for redshift (cf. Eq. 18) by means of an alternative argument.

Consider two light rays emitted at $t$ and $t+\delta t$ by the very same source. Suppose they are received by the same detector at time $t_{0}$ and $t_{0}+d t_{0}$. The geodesic equation of the first ray is

$$
t_{1}=t(\chi)
$$

while that of the second ray is

$$
t_{2}=t(\chi)+\delta t(\chi)
$$

It is clear that they are both solutions of the geodesic equation with the boundary conditions $t_{1}(\chi)=t(\chi), t_{1}(0)=t_{0}$, $\delta t(\chi)=\delta t, \delta t(0)=\delta t_{0}$ and $t_{2}(0)=t_{0}+\delta t_{0}$. By inserting them into (16) one gets

$$
\begin{aligned}
\frac{d t(\chi)}{d \chi} & =-\alpha(\chi, t(\chi)) \\
\frac{d \delta t(\chi)}{d \chi} & =-\delta t(\chi) \dot{\alpha}(\chi, t(\chi)) .
\end{aligned}
$$

The second equation describes the time dilation and can be straightforwardly solved to give

$$
\frac{\delta t_{0}}{\delta t}=e^{\int_{0}^{\chi} \dot{\alpha}(\chi, t(\chi)) d \chi}
$$

where the integral is calculated along the null geodesic of the photon. Since Eq. 18, can be rewritten as

$$
1+z=e^{\int_{0}^{\chi} \dot{\alpha}(\chi, t(\chi)) d \chi}
$$

we deduce that

$$
1+z=\frac{\delta t_{0}}{\delta t}
$$

[1] E. Milne, Relativity, Gravitation and World-structure, International series of monographs on physics (Clarendon Press, 1935), URL https://books .google.fr/books?id= QdCaswEACAA]

[2] E. Harrison, Cosmology: The Science of the Universe (Cambridge University Press, 2000), ISBN 9781139643450, URL https://books.google.fr/books?id=eFchAwAAQBA]

[3] R. Maartens, Philosophical Transactions of the Royal Society of London Series A 369, 5115 (2011), 1104.1300.

\section{Appendix C: Numerical redshift drift}

The numerical algorithm for calculating the redshift drift (referenced as method 3 in Sec. V) is here briefly discussed. The null-geodesics of a photon (labelled by the index 1) emitted at time $t$ and received at time $t_{0}$ by two galactic sources is parametrized by the equations

$$
\begin{aligned}
& z_{1}=z(\chi) \\
& t_{1}=t(\chi)
\end{aligned}
$$

as a function of the radial comoving coordinate $\chi$. A second photon, emitted and received by the same sources at the later times $\delta t$ and $\delta t_{0}$, will move along the path

$$
\begin{aligned}
& z_{2}=z(\chi)+\delta z(\chi) \\
& t_{2}=t(\chi)+\delta t(\chi)
\end{aligned}
$$

where the following boundary conditions are assumed

$$
\begin{aligned}
t(0) & =t_{0}, \\
z(0) & =0, \\
\delta z(0) & =0, \\
\delta t(0) & =\delta t_{0} .
\end{aligned}
$$

By substituting the photon path $\sqrt{C 1}$ and $(C 2)$ into the equations for the redshift (20) and 21) one gets, at leading order,

$$
\begin{aligned}
& \frac{d \delta z}{d \chi}=\dot{\alpha}(\chi, t(\chi)) \delta z+(1+z) \ddot{\alpha}(\chi, t(\chi)) \delta t \\
& \frac{d \delta t}{d \chi}=-\dot{\alpha}(\chi, t(\chi)) \delta t
\end{aligned}
$$

which, once solved along the geodesic $t_{1}=t\left(\chi, t_{0}\right)$, provide a numerical estimate of the redshift drift.
[4] J.-P. Uzan, C. Clarkson, and G. F. R. Ellis, Phys. Rev. Lett. 100, 191303 (2008), 0801.0068.

[5] C. Marinoni, J. Bel, and A. Buzzi, JCAP 2012, 036 (2012), 1205.3309.

[6] W. Valkenburg, V. Marra, and C. Clarkson, Mon. Not. Roy. Astron. Soc. 438, L6 (2014), 1209.4078.

[7] Z.-S. Zhang, T.-J. Zhang, H. Wang, and C. Ma, Phys. Rev. D 91, 063506 (2015), 1210.1775.

[8] C.-G. Park, H. Hyun, H. Noh, and J.-c. Hwang, 
Mon. Not. Roy. Astron. Soc. 469, 1924 (2017), 1611.02139.

[9] S. Sarkar, B. Pandey, and R. Khatri, Mon. Not. Roy. Astron. Soc. 483, 2453 (2019), 1810.07410.

[10] R. Jimenez, R. Maartens, A. R. Khalifeh, R. R. Caldwell, A. F. Heavens, and L. Verde, Journal of Cosmology and Astroparticle Physics 2019, 048 (2019), ISSN 1475-7516, URL http://dx. doi.org/10.1088/1475-7516/2019/05/048

[11] D. Camarena, V. Marra, Z. Sakr, and C. Clarkson (2021), 2107.02296.

[12] A. Krasinski, Inhomogeneous Cosmological Models (1997).

[13] G. Lemaître, Annales de la Soci\&eacute;t\&eacute; Scientifique de Bruxelles 53, 51 (1933).

[14] R. C. Tolman, Proceedings of the National Academy of Science 20, 169 (1934).

[15] H. Bondi, Mon. Not. Roy. Astron. Soc. 107, 410 (1947).

[16] K. Enqvist, General Relativity and Gravitation 40, 451 (2008), 0709.2044.

[17] V. Marra and A. Notari, Classical and Quantum Gravity 28, 164004 (2011), 1102.1015.

[18] M.-N. Célérier, Do we really see a cosmological constant in the supernovae data? (1999), astro-ph/9907206.

[19] K. Tomita, Astrophys. J. 529, 38 (2000), astro-ph/9906027.

[20] K. Enqvist and T. Mattsson, JCAP 2007, 019 (2007), astro$\mathrm{ph} / 0609120$.

[21] J. Garcia-Bellido and T. Haugbølle, JCAP 2008, 003 (2008), 0802.1523

[22] T. Biswas, A. Notari, and W. Valkenburg, JCAP 2010, 030 (2010), 1007.3065.

[23] M. Redlich, K. Bolejko, S. Meyer, G. F. Lewis, and M. Bartelmann, Astronomy \& Astrophysics 570, A63 (2014), 1408.1872.

[24] C. Stahl, International Journal of Modern Physics D 25, 1650066-67 (2016).

[25] M. Tokutake, K. Ichiki, and C.-M. Yoo, JCAP 2018, 033 (2018), 1712.04229.

[26] R. C. Keenan, A. J. Barger, and L. L. Cowie, Astrophys. J. 775, 62 (2013), 1304.2884.

[27] T. Shanks, L. M. Hogarth, N. Metcalfe, and J. Whitbourn, Mon. Not. Roy. Astron. Soc. 490, 4715 (2019), 1909.01878.

[28] V. V. Luković, B. S. Haridasu, and N. Vittorio, Mon. Not. Roy. Astron. Soc. 491, 2075 (2020), 1907.11219.

[29] N. Mustapha, C. Hellaby, and G. F. R. Ellis, Mon. Not. Roy. Astron. Soc. 292, 817 (1997), gr-qc/9808079.

[30] P. Sundell, E. Mörtsell, and I. Vilja, JCAP 2015, 037 (2015), 1503.08045 .

[31] A. Sandage, Astrophys. J. 136, 319 (1962).

[32] A. Loeb, Astrophys. J. Lett. 499, L111 (1998), astro$\mathrm{ph} / 9802122$.

[33] A. G. Kim, E. V. Linder, J. Edelstein, and D. Erskine, Astroparticle Physics 62, 195 (2015), 1402.6614.

[34] M. Visser, Gen. Rel. Grav. 37, 1541 (2005), gr-qc/0411131.

[35] J. Liske, A. Grazian, E. Vanzella, M. Dessauges, M. Viel, L. Pasquini, M. Haehnelt, S. Cristiani, F. Pepe, G. Avila, et al., Mon. Not. Roy. Astron. Soc. 386, 1192 (2008), 0802.1532.

[36] M. Martinelli, S. Pandolfi, C. J. A. P. Martins, and P. E. Vielzeuf, Phys. Rev. D 86, 123001 (2012), 1210.7166.

[37] H. R. Klöckner, D. Obreschkow, C. Martins, A. Raccanelli, D. Champion, A. Roy, A. Lobanov, J. Wagner, and R. Keller, Real time cosmology - a direct measure of the expansion rate of the universe (2015), 1501.03822.

[38] H.-R. Yu, T.-J. Zhang, and U.-L. Pen, Phys. Rev. Lett. 113, 041303 (2014), URL https://link . aps .org/doi/10. 1103/PhysRevLett. 113.041303

[39] P.-S. Corasaniti, D. Huterer, and A. r. Melchiorri, Phys. Rev. D 75, 062001 (2007), astro-ph/0701433.
[40] A. Balbi and C. Quercellini, Mon. Not. Roy. Astron. Soc. 382, 1623 (2007), 0704.2350.

[41] H. Zhang, W. Zhong, Z.-H. Zhu, and S. He, Phys. Rev. D 76, 123508 (2007), 0705.4409.

[42] J.-J. Geng, J.-F. Zhang, and X. Zhang, JCAP 2014, 018 (2014), 1407.7123.

[43] T. Denkiewicz, M. P. Dabrowski, C. J. A. P. Martins, and P. E. Vielzeuf, Phys. Rev. D 89, 083514 (2014), 1402.0520.

[44] C. J. A. P. Martins, M. Martinelli, E. Calabrese, and M. P. L. P. Ramos, Phys. Rev. D 94, 043001 (2016), 1606.07261.

[45] R.-Y. Guo and X. Zhang, European Physical Journal C 76, 163 (2016), 1512.07703.

[46] R. Lazkoz, I. Leanizbarrutia, and V. Salzano, European Physical Journal C 78, 11 (2018), 1712.07555.

[47] C. S. Alves, A. C. O. Leite, C. J. A. P. Martins, J. G. B. Matos, and T. A. Silva, Mon. Not. Roy. Astron. Soc. 488, 3607 (2019), 1907.05151.

[48] M. Korzyński and J. Kopiński, Journal of Cosmology and Astroparticle Physics 2018, 012 (2018), ISSN 1475-7516, URL http://dx.doi .org/10.1088/1475-7516/2018/03/012

[49] A. Heinesen, Physical Review D 103 (2021), ISSN 24700029, URL http://dx.doi .org/10.1103/PhysRevD . 103. Q23537

[50] A. Balcerzak and M. P. Dabrowski, Phys. Rev. D 87, 063506 (2013), 1210.6331.

[51] P. Mishra, M.-N. Célérier, and T. P. Singh, Phys. Rev. D 86, 083520 (2012), 1206.6026.

[52] S. M. Koksbang and S. Hannestad, JCAP 2016, 009 (2016), 1512.05624.

[53] C.-M. Yoo, T. Kai, and K.-I. Nakao, Phys. Rev. D 83, 043527 (2011), 1010.0091.

[54] C. Quercellini, L. Amendola, A. Balbi, P. Cabella, and M. Quartin, Phys. Rep. 521, 95 (2012), 1011.2646.

[55] Planck Collaboration, N. Aghanim, Y. Akrami, M. Ashdown, J. Aumont, C. Baccigalupi, M. Ballardini, A. J. Banday, R. B. Barreiro, N. Bartolo, et al., arXiv e-prints arXiv:1807.06209 (2018), 1807.06209.

[56] J. Kristian and R. K. Sachs, Astrophys. J. 143, 379 (1966).

[57] K. Lake, Phys. Rev. D 76, 063508 (2007), astro-ph/0703810.

[58] P. Dunsby, N. Goheer, B. Osano, and J.-P. Uzan, Journal of Cosmology and Astroparticle Physics 2010, 017 (2010), ISSN 1475-7516, URL http://dx.doi.org/10. 1088/1475-7516/2010/06/017.

[59] J.-P. Uzan, F. Bernardeau, and Y. Mellier, Phys. Rev. D 77, 021301 (2008), 0711.1950.

[60] J. Bel and C. Marinoni, Phys. Rev. Lett. 121, 021101 (2018), 1802.04495

[61] J. Silk, Astronomy \& Astrophysics 59, 53 (1977).

[62] X.-P. Yan, D.-Z. Liu, and H. Wei, Physics Letters B 742 (2015).

[63] C. Z. Vargas, F. T. Falciano, and R. R. R. Reis, Classical and Quantum Gravity 34, 025002 (2017).

[64] T. Clifton, P. G. Ferreira, and K. Land, Physical Review Letters 101 (2008), ISSN 1079-7114, URL http://dx . doi . org/10. 1103/PhysRevLett. 101.131302

[65] G. F. R. Ellis, General Relativity and Gravitation 41, 581 (2009), URL https://doi.org/10.1007/ S10714-009-0760-7

[66] L. Pèrenon, F. Piazza, C. Marinoni, and L. Hui, JCAP 2015, 029 (2015), 1506.03047.

[67] C. Quercellini, M. Quartin, and L. Amendola, Phys. Rev. Lett. 102, 151302 (2009), 0809.3675.

[68] A. Heinesen, Physical Review D 103 (2021), ISSN 24700029, URL http://dx.doi .org/10.1103/PhysRevD . 103. L081302 
[69] K. Bolejko, C. Wang, and G. F. Lewis, arXiv e-prints arXiv:1907.04495 (2019), 1907.04495. 